\title{
SPECIES DIVERSITY AND SUCCESSIONAL DYNAMICS IN THE SECONDARY FOREST OF OBAFEMI AWOLOWO UNIVERSITY BIOLOGICAL GARDENS ILE-IFE, NIGERIA
}

\author{
Nelson O. Uwalaka*, Joseph I. Muoghalu, Afolabi O. Osewole \\ Obafemi Awolowo University, Nigeria \\ *e-mail: uwalakanelson@gmail.com
}

Received: 01.08.2017

\begin{abstract}
Forests worldwide are being continuously disturbed by human activities and natural events, and tropical rainforest disturbance is specifically pronounced as indicated by its low biodiversity status. This study examined the floristic composition, species diversity and evenness in seven sample plots $\left(100,200,400\right.$ and $\left.2500 \mathrm{~m}^{2}\right)$ in a secondary forest of different ages in the Biological Gardens of the Obafemi Awolowo University, Ile-Ife. The aim of this study was to determine changes in these parameters with succession. In each sample plot, all vascular plant species were accounted for and enumerated. The data collected were used to establish the floristic composition, density, diversity and evenness of plant species in the forest. There were 85 woody species (69\%), 3 grass species (2\%), 13 forb species (11\%) and 21 climber species (17\%) in the forest. These concern 113 genera and 52 families. The number of woody species in the plots varied with the age of the plots, however, there were no clear trends in the density and diversity of species with plot age. The forest was dominated by early successional species (50\%) against the $33 \%$ contributed by the late successional species. Also, the youngest plot (Plot I) was dominated by early successional species while the older plots were dominated by a mixture of both early and late successional species. The similarity between plots during succession in the tropical forest was higher between plots of the same age. Also, this study showed that because of the directional pattern of succession, the species that converge to make up the diversity of forest of ages under consideration may be predictive of its recovery.
\end{abstract}

Key words: diversity, evenness, plant succession, secondary forest, similarity, successional species

\section{Introduction}

Plant succession has often been idealised as a process whereby a community that has suffered an episodic disturbance slowly regenerates a semblance of its former self in the absence of further disturbances (Horn, 1981). It is also viewed as an orderly unidirectional process of community change in which communities replace each other sequentially until a stable community is reached (Allaby, 2010). Forests worldwide are being continuously disturbed by human activities and natural events, and tropical rainforest disturbance is specifically pronounced as indicated by the low biodiversity status of tropical forests worldwide and the decreasing landmass areas of tropical rainforests. This disturbance drastically alters the community in the disturbed area, and causes it to transform to an earlier successional stage. The community then undergoes successional processes which lead it towards a climax community. Forest disturbance is now pronounced in tropical rainforests. This study focused on secondary forest succession, which is defined as the long-term directional change in community composition following a disturbance event, often at a large spatial scale (Chazdon, 2008). Some studies have demonstrated that in tropical forest fragments, the disturbances, whether natural or anthropogenic (Sheil \& Burslem, 2003) are critical in determining the dynamics of tree communities. This is because they alter resource availability (Denslow et al., 1998) and lead to differential mortality and recruitment rates of some species (Laurance et al., 1998). Therefore, secondary forest succession can be visualised as a continuum from an early stage where the factors that govern colonisation are most important (e.g., substrate conditions for germination, timing of seed arrival via off-site dispersal, presence of soilstored seeds and re-sprouts), to later stages where competitive ability and environmental requirements of species (determined primarily by species-specific growth rates, longevity, maximum size at maturity, degree of shade tolerance) largely dictate patterns of species replacement over time (Walker \& Chapin, 1987).

The African rain forest is recovering from past disturbance (Van Gemerden et al., 2003). Disturbance is believed to be one of the main factors influencing variations in species diversity (Connell, 1978; Noss, 1996). It has been hypothesised that in communities where post-disturbance succession is not driven by competitive regimes, disturbance intensity and frequency may have little effect on species richness (Chesson \& Huntly, 1997). Many 
chronosequence studies have also documented rapid recovery of species richness and species diversity during tropical forest succession, but these trends are strongly influenced by soil fertility and land use history (Guariguata \& Ostertag, 2001; Chazdon, 2003; Chazdon et al., 2007).

Although we do not know yet what processes influence the rate of change of species richness during tropical wet forest succession, three factors are probably involved. First, long-living pioneer species persist well into the understorey re-initiation stage, pre-empting space and slowing the rate of species turnover. Second, low light availability in young and intermediate aged old-growth forests and the rarity or absence of canopy gaps may restrict establishment and recruitment of gap-requiring tree species (Dupuy \& Chazdon, 2006). Third, low seed availability may limit colonisation of tree species (Chazdon, 2008).

This study was focused on tropical forest succession in a relatively small area where factors such as soil fertility and land use history are similar, thus facilitating a more in depth assessment of the successional pattern without these confounding factors. The secondary forest in the Biological Gardens and other parts of the Obafemi Awolowo University, Ile-Ife campus are constantly disturbed either by clearing, farming, establishment of infrastructural facilities or fuelwood gathering. Knowledge on the recovery pattern of tropical forests from previous perturbations is important in the conservation of its rich biodiversity. This knowledge is necessary in order to halt the current increasing rate of forest disturbance in the Biological Gardens. We addressed the following questions: (1) Do forests of similar ages vary in density and diversity? (2) Do forests of similar ages show the same pattern of species similarity? (3) Do forests of similar ages show similar convergence in species abundance? To answer these questions, the successional processes in disturbed areas of different successional ages in the Biological Gardens and a mature community were studied, and comparisons made in terms of species composition and structure. The aim of this was to determine the species richness and diversity of these disturbed areas of different ages as well as their similarity in floristic and structural characteristics.

\section{Material and Methods}

\section{Study area}

Ile-Ife lies in the lowland forest zone (Keay, 1959; Charter, 1969; White, 1983). The eleven most abundant plant species are Albizia zygia (DC.) J.F.
Macbr., Blighia unijugata Baker, Bombax buonopozense P. Beauv., Bosqueia angolensis Ficalho, Commiphora kerstingii Engl., Elaeis guineensis Jacq., Ficus mucuso Welw. ex Ficalho, Funtumia elastica (Preuss) Stapf, Holarrhena floribunda (G. Don) T. Durand \& Schinz, Manihot glaziovii Müll. Arg. and Pycnanthus angolensis (Welw.) Warb. (Isichei et al., 1986). The most frequently occurring plant families are Apocynaceae, Euphorbiaceae, Mimosaceae, Moraceae, Rubiaceae, and Sapindaceae. There are two prominent seasons in Ile-Ife area, the rainy season (March to November) and the dry season (November to March). The most recent climatic survey conducted in 2013 by the Atmospheric Physics Research Group, Department of Physics and Engineering Physics, Obafemi Awolowo University, Ile-Ife, showed that the mean annual rainfall at Ile-Ife averaged $1302 \mathrm{~mm}$ per year, with relative humidity of $82.8 \%$, average temperature of $25.5^{\circ} \mathrm{C}$, solar radiation of 164.30 $\mathrm{Wm}^{-2}$ and average wind speed of $2.06 \mathrm{~km} / \mathrm{h}$.

The soil has been classified as Lixisols (FAO/ UNESCO, 1974) and ultisols (USDA, 1975). The soils, which are usually acidic, contain less than $10 \%$ clay which is mainly kaolinite and hence are characterised by low cation exchange capacity and low water-holding capacity (Ayodele, 1986).

The study was carried out within $0.205 \mathrm{~km}^{2} \mathrm{sec}-$ ondary forest in the Obafemi Awolowo University Biological Gardens, Ile-Ife, Nigeria (Fig.). The forest, which was ravaged by a ground fire 34 years ago (in 1983), is a natural regrowth under conservation within and outside the Gardens with minimal human disturbance. Before and after the incidence of the ground fire, some permanent sampling sites were marked out (Isichei et al., 1986), on which several natural regeneration monitoring studies have been conducted on different plant life forms (trees, shrubs, herbs and climbers). A permanent sampling plot $\left(0.0025 \mathrm{~km}^{2}\right)$ marked out before the incidence of the ground fire, which was not affected by the ground fire (termed an unburnt plot), was used as a means of comparison in terms of floristic and structural characteristics. Seven plots, between latitudes $7^{\circ} 31.33^{\prime} \mathrm{N}-7^{\circ} 31.45^{\prime} \mathrm{N}$ and longitudes $4^{\circ} 31.42^{\prime} \mathrm{E}$ $-4^{\circ} 31.46^{\prime} \mathrm{E}$ (Table 1 ) undergoing succession of different ages were randomly selected in the Gardens for the study. The altitudes of the plots ranged from $286 \mathrm{~m}$ to $312 \mathrm{~m}$ above sea level (a.s.1.), with plot VII having the highest altitude ( $312 \mathrm{~m}$ a.s.1.). The ages of the plots ranged from 5-70 years (Table 1). The ages of the plots was estimated since the last time of stand disturbance. 


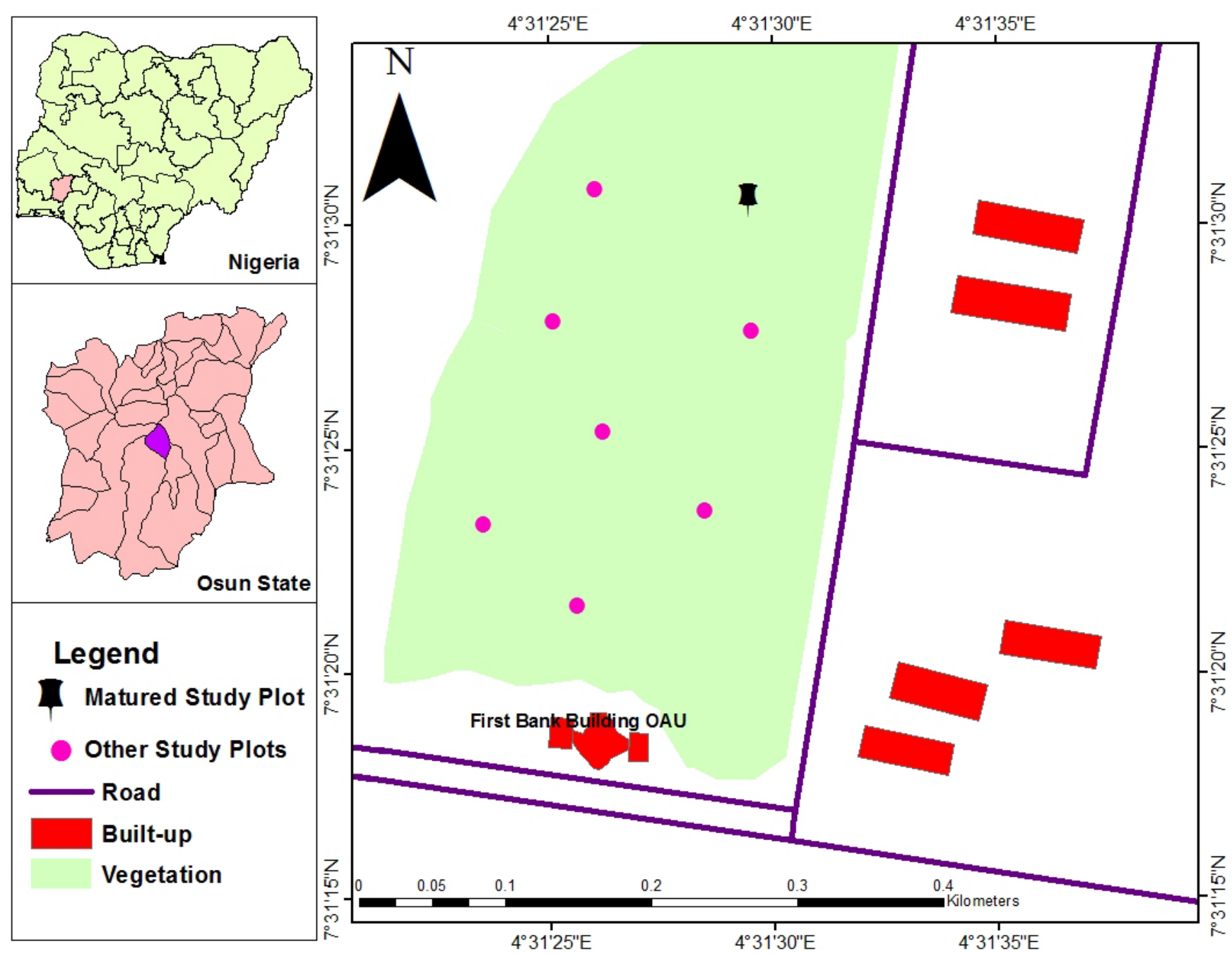

Fig. Map of the Biological Gardens of Obafemi Awolowo University, Ile-Ife, with the sampling locations where the study was carried out.

The vegetation of the plots was open except for the presence of some mature tree species and some regrowths that have colonised the cleared areas. Many growth forms including woody species, herbs, climbers, and some mosses growing on decaying felled wood were present in the plots.

\section{Data collection}

Seven plots of different sizes depending on the size of the disturbed areas within different parts of the secondary forest in the Biological Gardens were marked out using a measuring tape. Five of the sample plots, named plots I, III, IV, V, VI, were $20 \times 20 \mathrm{~m}$ in size, one plot (plot II) was $15 \times 15 \mathrm{~m}$ and plot VII was $10 \times 10 \mathrm{~m}$. A more mature plot (plot VIII: $50 \times 50 \mathrm{~m}$ ) which has undergone a series of successional stages, in terms of species composition, diversity and evenness (Ogunniran \& Muoghalu, 2007), was used as a plot with which the selected plots were compared. In each plot, all the plant species were determined. Specimens of plant species that could not be identified in the field were collected and identified in the IFE Herbarium. From the data obtained in the field, the following vegetation attributes were determined for each plot: species composition, density $\left(\mathrm{ha}^{-1}\right)$, Shannon-Weiner diversity index $\left(\mathrm{H}^{\prime}\right)$, evenness of distribution of species and Sørensen's index of similarity between the plots.

\section{Data analysis}

The species composition of the plots was established by listing all the species encountered in each plot and summing up to get the total number of plant species for a plot. The relative abundance of species per plot was used to compile a list of dominant woody species and their successional status was determined according to the results of Ademoh et al. (2017). Species with high relative abundance were termed dominant. 
Table 1. Geographic location and ages of the study plots after disturbance

\begin{tabular}{ccccc}
\hline PLOT & LATITUDE $(\mathrm{N})$ & LONGITUDE $(\mathrm{E})$ & ALTITUDE $(\mathrm{m}$ a.s.l.) & AGE OF PLOT \\
\hline I & $7^{\circ} 31.332^{\prime}$ & $4^{\circ} 31.423^{\prime}$ & 296 & 5 years \\
II & $7^{\circ} 31.354^{\prime}$ & $4^{\circ} 31.444^{\prime}$ & 288 & 34 years \\
III & $7^{\circ} 31.423^{\prime}$ & $4^{\circ} 31.446^{\prime}$ & 291 & 34 years \\
IV & $7^{\circ} 31.456^{\prime}$ & $4^{\circ} 31.463^{\prime}$ & 297 & 34 years \\
V & $7^{\circ} 31.459^{\prime}$ & $4^{\circ} 31.444^{\prime}$ & 300 & 34 years \\
VI & $7^{\circ} 31.471^{\prime}$ & $4^{\circ} 31.460^{\prime}$ & 292 & 34 years \\
VII & $7^{\circ} 31.456^{\prime}$ & $4^{\circ} 31.411^{\prime}$ & 312 & 34 years \\
VIII & $7^{\circ} 31.360^{\prime}$ & $4^{\circ} 31.480^{\prime}$ & 286 & $>70$ years \\
\hline
\end{tabular}

To estimate the convergence of species during succession, the similarity in species composition in each plot were compared using the Sørensen index of similarity (ISS). The comparison done by the measurement of the similarity and dissimilarity between sampling plot pairs was based on the presence or absence of species of interest in each study plot. The same method was used to estimate the convergence of species in the unburnt plot (Plot VIII) and other plots (Plots I-VII). Values near 1 indicate nearly identical community composition between the pair of plots and values near 0 indicates that communities have very little compositional overlap between the plot pairs. The Sørensen similarity index was calculated as: $I S S=\frac{2 C}{A+B}$. Where: $\mathrm{C}$ is the number of species common to plots $\mathrm{A}$ and $\mathrm{B}$; $\mathrm{A}$ is the number of species in plot $\mathrm{A} ; \mathrm{B}$ is the number of species in plot B.

The woody plant diversity was calculated using the Shannon-Weiner diversity index as: $H^{1}=-\sum P_{i} \ln \left(P_{i}\right)$ . Where: $\mathrm{H}^{\prime}$ is the Shannon-Weiner diversity index; $P_{i}=\frac{i}{N}$, the relative abundance of each species $\langle i \mathrm{i}\rangle ; \mathrm{N}$ is the total number of individuals of all species; $n_{i}$ is the number of individuals of species.

The evenness of distribution of species was calculated using the Pielou's evenness index, J: $J=\frac{H^{1}}{\ln (S)}$. Where: $J$ is the Pielou's evenness index; $\mathrm{H}^{\prime}$ is the Shannon-Weiner diversity index; $\mathrm{S}$ is the total number of species on each plot. The estimation of the density, diversity and evenness indices all accounted for unequal plot sizes.

\section{Results}

Floristic composition and successional status of woody species in the plots

The plots contained a total of 123 plant species within 113 genera and 52 families (Table 2). Woody species were responsible for 69\% (85 species), grasses for 3\% (3 species), forbs for $11 \%$ (13 species) and climbers for $17 \%$ (21 species) of the plant communities. The highest number of forb and grass species (6) was found in the youngest plot (plot I), followed by plot IV, which had 6 forb species (Table 3).

Plot I, the youngest plot, and plot VII, contained the least number of woody species, each with 15 species, while the highest number of woody species (48) was found in the oldest plot (plot VIII) (Table 3). Plot I (the youngest plot) and plot VI had the highest number of grass species (2 each). However, grass species were virtually absent in plot IV, V, VII, and VIII (Table 3). Climbers were most widely found in the plots having the highest number of woody species (plot II, IV, V, VI, VII and VIII). The most common woody species families in the plots were Apocynaceae, Bignoniaceae, Euphorbiaceae, Papilionaceae, Moraceae, and Sterculiaceae, while the commonest species were Cola millenii K. Schum., Ficus mucoso Welw., Mallotus oppositifolius (Geiseler) Mull. Arg., and Newbouldia laevis (P. Beauv.) Seem. ex Bureau (Table 2). The number of plant species in each plot varied with the age of the plots.

This trend was also observed in the total number of species in the plots (Table 3), with the youngest plot and some older plots (plot I, VI and VII) having the lowest number of all species. In plot III, there was an abundant number of Bambusa vulgaris Schrad. ex Wendel., which suppressed the growth of many woody species and as such, the woody species found were thin-stemmed and short.

In terms of dominance, the youngest plot (Plot I) comprised mostly early successional species while the other plots had a mixture of both early and late successional species (Table 4). Among the 30 dominant woody species in the forest, 33\% were late successional species, 50\% were early successional species while the status of $17 \%$ of the species could not be determined. 
Table 2. Floristic composition of plant species in the study plots in the Biological Gardens of Obafemi Awolowo University, Ile-Ife, Nigeria

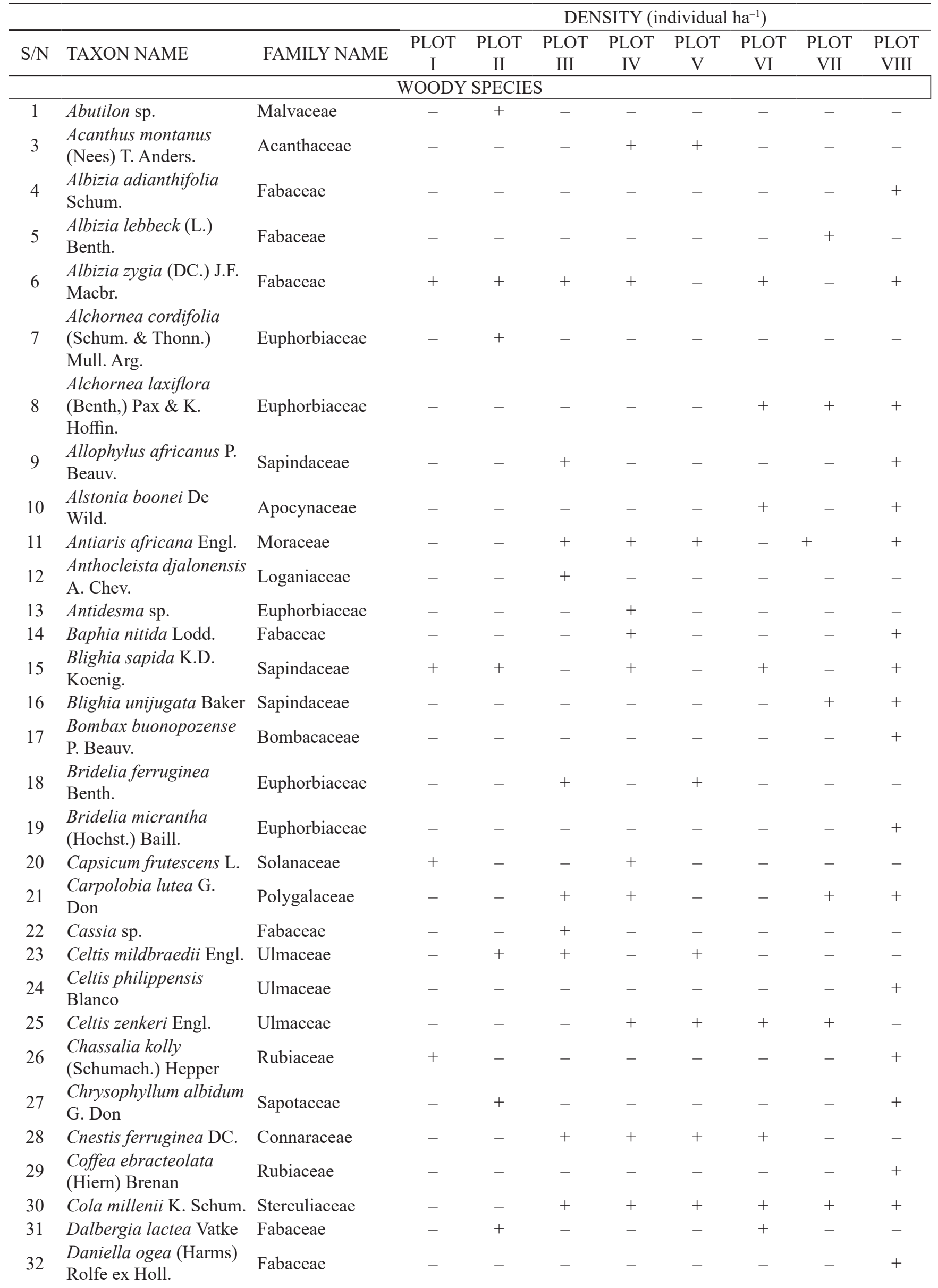




\begin{tabular}{|c|c|c|c|c|c|c|c|c|c|c|}
\hline \multirow[b]{2}{*}{$\mathrm{S} / \mathrm{N}$} & \multirow[b]{2}{*}{ TAXON NAME } & \multirow[b]{2}{*}{ FAMILY NAME } & \multicolumn{8}{|c|}{ DENSITY (individual ha ${ }^{-1}$ ) } \\
\hline & & & $\begin{array}{c}\text { PLOT } \\
\text { I }\end{array}$ & $\begin{array}{l}\text { PLOT } \\
\text { II }\end{array}$ & $\begin{array}{l}\text { PLOT } \\
\text { III }\end{array}$ & $\begin{array}{l}\text { PLOT } \\
\text { IV }\end{array}$ & $\begin{array}{c}\text { PLOT } \\
\mathrm{V}\end{array}$ & $\begin{array}{l}\text { PLOT } \\
\text { VI }\end{array}$ & $\begin{array}{c}\text { PLOT } \\
\text { VII }\end{array}$ & $\begin{array}{c}\text { PLOT } \\
\text { VIII }\end{array}$ \\
\hline 33 & $\begin{array}{l}\text { Deinbollia pinna- } \\
\text { ta (Poir.) Schum. \& } \\
\text { Thonn. }\end{array}$ & Sapindaceae & - & + & - & + & + & - & - & + \\
\hline 34 & $\begin{array}{l}\text { Dialium guineense } \\
\text { Willd. }\end{array}$ & Fabaceae & - & - & - & - & - & - & + & - \\
\hline 35 & $\begin{array}{l}\text { Diospyros monbuttensis } \\
\text { Gurke }\end{array}$ & Ebenaceae & - & + & - & - & + & - & - & + \\
\hline 36 & $\begin{array}{l}\text { Dracaena arborea } \\
\text { (Willd.) Link }\end{array}$ & Dracaenaceae & - & - & - & - & - & - & - & + \\
\hline 37 & Drypetes sp. & Euphorbiaceae & - & - & - & - & + & - & - & - \\
\hline 38 & Elaeis guineensis Jacq. & Aracaceae & - & - & + & - & - & - & - & - \\
\hline 39 & $\begin{array}{l}\text { Fagara leprieurii } \\
\text { (Guill. \& Perr.) Engl. }\end{array}$ & Rutaceae & - & - & - & + & - & - & - & - \\
\hline 40 & Ficus exasperata Vahl. & Moraceae & + & - & - & - & - & - & - & - \\
\hline 41 & Ficus mucoso Welw. & Moraceae & + & + & + & + & + & - & - & + \\
\hline 42 & $\begin{array}{l}\text { Funtumia elastica } \\
\text { (Preuss) Stapf. }\end{array}$ & Apocynaceae & - & - & + & + & + & + & - & + \\
\hline 43 & $\begin{array}{l}\text { Glyphaea brevis } \\
\text { (Spreng.) Monach. }\end{array}$ & Tiliaceae & - & - & + & - & - & - & + & + \\
\hline 44 & $\begin{array}{l}\text { Hedranthera barteri } \\
\text { (Hook. f.) Pichon } \\
\text { Holarrhena floribunda }\end{array}$ & Apocynaceae & - & - & + & + & - & + & - & - \\
\hline 45 & $\begin{array}{l}\text { (G. Don) T. Durand \& } \\
\text { Schinz }\end{array}$ & Apocynaceae & - & + & + & - & + & - & - & + \\
\hline 46 & $\begin{array}{l}\text { Homalium letestui } \\
\text { Pellegr. }\end{array}$ & Samydaceae & + & - & - & - & - & - & - & - \\
\hline 47 & Icacina trichantha Oliv. & Icacinaceae & - & + & - & + & + & - & - & - \\
\hline 48 & $\begin{array}{l}\text { Lecaniodiscus } \\
\text { cupanioides Planch. }\end{array}$ & Sapindaceae & - & - & - & + & + & - & - & + \\
\hline 49 & $\begin{array}{l}\text { Microdesmis puberula } \\
\text { Hook. f. ex Planch. }\end{array}$ & Pandaceae & - & - & + & + & + & - & - & + \\
\hline 50 & $\begin{array}{l}\text { Mallotus oppositifolius } \\
\text { (Geiseler) Mull. Arg. }\end{array}$ & Euphorbiaceae & - & + & + & + & + & + & + & - \\
\hline 51 & $\begin{array}{l}\text { Manihot glaziovii Mull. } \\
\text { Arg. }\end{array}$ & Euphorbiaceae & - & + & - & - & - & - & - & + \\
\hline 52 & $\begin{array}{l}\text { Margaritaria discoidea } \\
\text { (Baill.) G.L. Webster }\end{array}$ & Euphorbiaceae & - & + & - & - & - & - & - & - \\
\hline 53 & $\begin{array}{l}\text { Milletia thonningii } \\
\text { (Schumach. \& Thonn.) } \\
\text { Baker }\end{array}$ & Fabaceae & - & - & - & - & + & - & - & + \\
\hline 54 & $\begin{array}{l}\text { Monodora tenuifolia } \\
\text { Benth. }\end{array}$ & Annonaceae & - & - & + & - & - & - & - & + \\
\hline 55 & Morinda lucida Benth. & Rubiaceae & - & - & - & - & - & - & - & + \\
\hline 56 & $\begin{array}{l}\text { Myrianthus arboreus P. } \\
\text { Beauv. }\end{array}$ & Moraceae & - & - & - & - & + & - & - & + \\
\hline 57 & $\begin{array}{l}\text { Napoleona imperialis } \mathrm{P} . \\
\text { Beauv. }\end{array}$ & Lecythidaceae & - & - & + & + & + & + & - & - \\
\hline 58 & $\begin{array}{l}\text { Napoleona vogelii } \\
\text { Hook. \& Planch. }\end{array}$ & Lecythidaceae & - & - & - & - & - & + & - & + \\
\hline 59 & $\begin{array}{l}\text { Newbouldia laevis } \\
\text { (P. Beauv.) Seem. ex } \\
\text { Bureau }\end{array}$ & Bignoniaceae & + & + & + & - & + & + & + & + \\
\hline 60 & Olax sp. & Olacaceae & - & - & - & - & + & + & - & - \\
\hline 61 & $\begin{array}{l}\text { Oxyanthus speciosus } \\
\text { DC. }\end{array}$ & Rubiaceae & - & - & - & - & - & - & - & + \\
\hline
\end{tabular}




\begin{tabular}{|c|c|c|c|c|c|c|c|c|c|c|}
\hline \multirow[b]{2}{*}{$\mathrm{S} / \mathrm{N}$} & \multirow[b]{2}{*}{ TAXON NAME } & \multirow[b]{2}{*}{ FAMILY NAME } & \multicolumn{8}{|c|}{ DENSITY (individual ha ${ }^{-1}$ ) } \\
\hline & & & $\begin{array}{l}\text { PLOT } \\
\text { I }\end{array}$ & $\begin{array}{l}\text { PLOT } \\
\text { II }\end{array}$ & $\begin{array}{l}\text { PLOT } \\
\text { III }\end{array}$ & $\begin{array}{l}\text { PLOT } \\
\text { IV }\end{array}$ & $\begin{array}{l}\text { PLOT } \\
\text { V }\end{array}$ & $\begin{array}{l}\text { PLOT } \\
\text { VI }\end{array}$ & $\begin{array}{l}\text { PLOT } \\
\text { VII }\end{array}$ & $\begin{array}{c}\text { PLOT } \\
\text { VIII }\end{array}$ \\
\hline 62 & $\begin{array}{l}\text { Pauridiantha hirtella } \\
\text { (Benth.) Bremek. }\end{array}$ & Rubiaceae & - & - & - & - & - & + & - & - \\
\hline 63 & $\begin{array}{l}\text { Pavetta corymbosa } \\
\text { (DC.) W.F. Williams } \\
\text { Phyllanthus }\end{array}$ & Rubiaceae & - & - & + & - & + & - & - & - \\
\hline 64 & $\begin{array}{l}\text { muellerianus (Kuntze) } \\
\text { Exell }\end{array}$ & Euphorbiaceae & + & - & + & - & - & - & - & - \\
\hline 65 & $\begin{array}{l}\text { Piliostigma thonningii } \\
\text { (Schumach.) Milne- } \\
\text { Redh. }\end{array}$ & Fabaceae & + & - & - & - & - & - & - & - \\
\hline 66 & $\begin{array}{l}\text { Piptadenastrum africa- } \\
\text { num (Hook. f.) Brenan }\end{array}$ & Fabaceae & - & - & - & - & + & - & - & + \\
\hline 67 & Psychotria sp. & Rubiaceae & - & - & + & - & - & - & - & - \\
\hline 68 & $\begin{array}{l}\text { Pterocarpus } \\
\text { mildbraedii Harms }\end{array}$ & Fabaceae & - & - & - & + & - & - & - & + \\
\hline 69 & $\begin{array}{l}\text { Pycnanthus angolensis } \\
\text { (Welw.) Warb. }\end{array}$ & Myristicaceae & + & - & - & + & - & - & - & + \\
\hline 70 & $\begin{array}{l}\text { Rauvolfia vomitoria } \\
\text { Afzel. }\end{array}$ & Apocynaceae & - & - & - & - & - & - & - & + \\
\hline 71 & $\begin{array}{l}\text { Ricinodendron } \\
\text { heudelotii (Baill.) Pierre } \\
\text { ex Pax }\end{array}$ & Euphorbiaceae & - & - & + & + & + & + & + & - \\
\hline 72 & Ritchiea sp. & Capparidaceae & - & - & - & + & - & - & - & - \\
\hline 73 & $\begin{array}{l}\text { Rothmannia longiflora } \\
\text { Salisb. }\end{array}$ & Rubiaceae & - & - & - & - & + & - & - & - \\
\hline 74 & $\begin{array}{l}\text { Securinega virosa } \\
\text { (Roxb. ex Willd.) Baill. }\end{array}$ & Euphorbiaceae & + & - & + & - & - & - & - & - \\
\hline 75 & $\begin{array}{l}\text { Solanum erianthum D. } \\
\text { Don }\end{array}$ & Solanaceae & + & - & - & - & - & - & - & - \\
\hline 76 & $\begin{array}{l}\text { Sorindeia warneckei } \\
\text { Engl. }\end{array}$ & Anarcadiaceae & - & + & - & - & - & + & - & - \\
\hline 77 & $\begin{array}{l}\text { Sphenocentrum } \\
\text { jollyanum Pierre }\end{array}$ & Sterculiaceae & - & - & + & + & + & + & - & + \\
\hline 78 & Spondias mombin L. & Anarcadiaceae & - & + & - & - & - & - & - & + \\
\hline 79 & $\begin{array}{l}\text { Sterculia tragacantha } \\
\text { Lindl. }\end{array}$ & Sterculiaceae & - & - & - & - & - & - & + & + \\
\hline 80 & $\begin{array}{l}\text { Tetrapleura tetraptera } \\
\text { (Schum. \& Thonn.) } \\
\text { Taub. }\end{array}$ & Fabaceae & - & - & - & - & - & - & - & + \\
\hline 81 & $\begin{array}{l}\text { Trema orientalis }(\mathrm{L} .) \\
\text { Blume }\end{array}$ & Ulmaceae & + & - & - & - & - & - & - & + \\
\hline 82 & $\begin{array}{l}\text { Trichilia heudelotii } \\
\text { Planch. ex Oliv. }\end{array}$ & Meliaceae & - & - & - & - & - & - & - & + \\
\hline 83 & $\begin{array}{l}\text { Trichilia prieureana A. } \\
\text { Juss. }\end{array}$ & Meliaceae & - & + & - & + & + & - & - & + \\
\hline 84 & $\begin{array}{l}\text { Trilepsium } \\
\text { madagascariense DC. }\end{array}$ & Moraceae & - & - & - & + & + & - & + & + \\
\hline 85 & $\begin{array}{l}\text { Voacanga africana } \\
\text { Stapf. }\end{array}$ & Apocynaceae & + & + & - & - & - & - & - & + \\
\hline \multicolumn{11}{|c|}{ FORB SPECIES } \\
\hline 1 & $\begin{array}{l}\text { Anchomanes difformis } \\
\text { (Blume) Engl. }\end{array}$ & Araceae & - & - & - & + & - & - & - & - \\
\hline 2 & $\begin{array}{l}\text { Asystasia gangetica (L.) } \\
\text { T. Anders }\end{array}$ & Acanthaceae & - & + & - & + & - & - & + & - \\
\hline 3 & Canna indica $\mathrm{L}$. & Cannaceae & + & - & - & - & - & - & - & - \\
\hline
\end{tabular}




\begin{tabular}{|c|c|c|c|c|c|c|c|c|c|c|}
\hline \multirow[b]{2}{*}{$\mathrm{S} / \mathrm{N}$} & \multirow[b]{2}{*}{ TAXON NAME } & \multirow[b]{2}{*}{ FAMILY NAME } & \multicolumn{8}{|c|}{ DENSITY (individual $\mathrm{ha}^{-1}$ ) } \\
\hline & & & $\begin{array}{l}\text { PLOT } \\
\text { I }\end{array}$ & $\begin{array}{l}\text { PLOT } \\
\text { II }\end{array}$ & $\begin{array}{l}\text { PLOT } \\
\text { III }\end{array}$ & $\begin{array}{l}\text { PLOT } \\
\text { IV }\end{array}$ & $\begin{array}{l}\text { PLOT } \\
\mathrm{V}\end{array}$ & $\begin{array}{l}\text { PLOT } \\
\text { VI }\end{array}$ & $\begin{array}{l}\text { PLOT } \\
\text { VII }\end{array}$ & $\begin{array}{c}\text { PLOT } \\
\text { VIII }\end{array}$ \\
\hline 4 & Carica papaya $\mathrm{L}$ & Caricaceae & + & + & - & - & - & - & - & - \\
\hline 5 & $\begin{array}{l}\text { Chromolaena odorata } \\
\text { (L.) R.M. King \& H. } \\
\text { Rob. }\end{array}$ & Asteraceae & + & - & - & + & - & - & - & - \\
\hline 6 & Commelina sp. & Commelinaceae & - & - & - & - & + & - & - & - \\
\hline 7 & Cyathula sp. & Amaranthaceae & - & + & - & + & - & - & - & - \\
\hline 8 & Maranthocleoa sp. & Maranthaceae & - & - & - & + & - & - & - & - \\
\hline 9 & Musa sapientum L. & Musaceae & - & - & - & + & - & - & - & - \\
\hline 10 & Petiveria alliacea $\mathrm{L}$. & Phytolaccaceae & - & + & + & - & - & - & - & - \\
\hline 11 & $\begin{array}{l}\text { Phaulopsis barteri (T. } \\
\text { Anders.) Lindau }\end{array}$ & Acanthaceae & + & - & - & - & - & - & - & - \\
\hline 12 & $\begin{array}{l}\text { Talinum triangulare } \\
\text { (Jacq.) Willd. }\end{array}$ & Portulacaceae & - & - & - & - & - & + & - & - \\
\hline \multicolumn{11}{|c|}{ GRASS SPECIES } \\
\hline 1 & $\begin{array}{l}\text { Bambusa vulgaris } \\
\text { Schrad. ex Wendel. }\end{array}$ & Poaceae & - & - & + & - & - & - & - & - \\
\hline 2 & $\begin{array}{l}\text { Digitaria exilis } \\
\text { (Kippist) Stapf. }\end{array}$ & Poaceae & + & - & - & - & - & + & - & - \\
\hline 3 & $\begin{array}{l}\text { Panicum maximum } \\
\text { Jacq. }\end{array}$ & Poaceae & + & + & - & - & - & + & - & - \\
\hline \multicolumn{11}{|c|}{ CLIMBER SPECIES } \\
\hline 1 & Abrus precatorius L. & Fabaceae & - & + & - & - & - & - & - & - \\
\hline 2 & $\begin{array}{l}\text { Acacia ataxacantha } \\
\text { DC. }\end{array}$ & Fabaceae & - & - & - & - & + & - & - & + \\
\hline 3 & Alafia barteri Oliv. & Apocynaceae & - & - & - & + & - & - & - & + \\
\hline 4 & Baissea sp. & Apocynaceae & - & + & - & + & + & - & + & + \\
\hline 5 & Cissus sp. & Ampelidaceae & + & + & - & + & + & - & - & - \\
\hline 6 & Combretum sp. & Combretaceae & + & + & + & + & + & + & + & - \\
\hline 7 & $\begin{array}{l}\text { Culcasia scandens } \mathrm{P} . \\
\text { Beauv. }\end{array}$ & Araceae & - & - & - & - & + & - & - & - \\
\hline 8 & Hippocratea sp. & Celastraceae & - & - & + & - & - & - & - & + \\
\hline 9 & Jateorhiza sp. & Menispermaceae & + & - & - & - & - & - & + & + \\
\hline 10 & $\begin{array}{l}\text { Leptoderris micrantha } \\
\text { Dunn. }\end{array}$ & Fabaceae & - & - & - & - & + & - & - & - \\
\hline 11 & Memecylon sp. & Melastomataceae & - & - & - & + & - & - & - & - \\
\hline 12 & $\begin{array}{l}\text { Mezoneuron } \\
\text { benthamianum Baill. }\end{array}$ & Fabaceae & - & - & - & - & - & - & + & + \\
\hline 13 & $\begin{array}{l}\text { Momordica charantia } \\
\text { L. }\end{array}$ & Cucurbitaceae & + & + & + & - & - & - & - & - \\
\hline 14 & $\begin{array}{l}\text { Mondia whitei (Hook. } \\
\text { f.) Skeels }\end{array}$ & Periplocaceae & - & + & - & - & - & - & - & - \\
\hline 15 & $\begin{array}{l}\text { Mussaenda eleganz } \\
\text { Schum. Thonn. }\end{array}$ & Rubiaceae & - & - & - & - & - & + & - & - \\
\hline 16 & $\begin{array}{l}\text { Parquetina nigrescens } \\
\text { (Afzel.) Bullock }\end{array}$ & Periplocaceae & + & - & - & - & - & - & - & - \\
\hline 17 & Paullinia pinnata L. & Sapindaceae & - & + & + & - & - & - & - & - \\
\hline 18 & Phyllantus sp. & Euphorbiaceae & - & - & - & - & - & - & + & - \\
\hline 19 & $\begin{array}{l}\text { Salacia erecta (G. Don) } \\
\text { Walp. }\end{array}$ & Celastraceae & - & - & - & - & - & - & + & - \\
\hline 20 & Sida urens L. & Malvaceae & + & - & - & - & - & - & - & - \\
\hline 21 & $\begin{array}{l}\text { Smilax kraussiana } \\
\text { Meisn. }\end{array}$ & Smilacaceae & - & - & + & - & - & + & - & - \\
\hline 22 & Thunbergia sp. & Acanthaceae & - & - & - & + & - & - & - & - \\
\hline
\end{tabular}

Key: + represents presence of species; - represents absence of species 
Table 3. Summary of floristics and structural parameters of vegetation of the study plots

\begin{tabular}{lcccccccc}
\hline \multicolumn{1}{c}{ Vegetation parameters } & $\begin{array}{c}\text { PLOT I } \\
\text { (5 years) }\end{array}$ & $\begin{array}{c}\text { PLOT II } \\
\text { (43 years) }\end{array}$ & $\begin{array}{c}\text { PLOT III } \\
\text { (41 years) }\end{array}$ & $\begin{array}{c}\text { PLOT IV } \\
\text { (41 years) }\end{array}$ & $\begin{array}{c}\text { PLOT V } \\
\text { (41 years) }\end{array}$ & $\begin{array}{c}\text { PLOT VI } \\
\text { (41 years) }\end{array}$ & $\begin{array}{c}\text { PLOT VII } \\
\text { (41 years) }\end{array}$ & $\begin{array}{c}\text { PLOT VIII } \\
\text { (>70 years) }\end{array}$ \\
\hline Number of tree and shrub species & 15 & 20 & 27 & 28 & 29 & 19 & 14 & 48 \\
Number of grass species & 2 & 1 & 1 & 0 & 0 & 2 & 0 & 0 \\
Number of forb species & 4 & 4 & 1 & 6 & 1 & 1 & 1 & 0 \\
Number of climber species & 6 & 7 & 5 & 6 & 6 & 3 & 6 & 6 \\
Total number of plant species & 27 & 32 & 34 & 40 & 36 & 25 & 21 & 54 \\
Number of plant genera & 26 & 32 & 33 & 40 & 35 & 24 & 21 & 41 \\
Number of plant family & 24 & 22 & 26 & 26 & 24 & 18 & 16 & 26 \\
Density of trees and shrubs (ha $\left.{ }^{-1}\right)$ & 3700 & 7466 & $46+$ & $29+$ & 3300 & 1950 & 3100 & 14288 \\
Shannon-Weiner species & 2.371 & 2.677 & 2.796 & 3.24 & 3.202 & 2.82 & 2.559 & 2.744 \\
diversity index (H') & & & & & & & & \\
Species evenness (J) & 0.474 & 0.523 & 0.508 & 0.678 & 0.656 & 0.647 & 0.745 & 0.709 \\
\hline
\end{tabular}

Table 4. List of dominant species and their successional status in the different study plots in the Biological Gardens of Obafemi Awolowo University, Ile-Ife, Nigeria

\begin{tabular}{|c|c|c|c|c|c|c|c|c|c|}
\hline \multirow{2}{*}{ Species name } & \multirow{2}{*}{ Successional status } & \multicolumn{8}{|c|}{ Plot } \\
\hline & & I & II & III & IV & $\mathrm{V}$ & VI & VII & VIII \\
\hline Albizia lebbeck & Early & - & - & - & - & - & - & + & - \\
\hline Albizia zygia & Early & - & + & + & - & - & - & - & - \\
\hline Alchornea cordifolia & Early & - & + & - & - & - & - & - & - \\
\hline Alchornea laxiflora & Early & - & - & - & - & - & + & - & + \\
\hline Alstonia boonei & Early & - & - & - & - & - & + & - & - \\
\hline Blighia sapida & Early & - & + & - & + & - & + & - & + \\
\hline Carpolobia lutea & Undetermined & - & - & - & + & - & - & - & + \\
\hline Celtis zenkeri & Early & - & - & - & + & - & - & + & - \\
\hline Cnestis ferruginea & Undetermined & - & - & - & + & - & - & - & - \\
\hline Cola millenii & Early & - & - & - & + & + & + & - & - \\
\hline Deinbollia pinnata & Late & - & + & - & + & - & - & - & + \\
\hline Ficus exasperata & Early & + & - & - & - & - & - & - & - \\
\hline Ficus mucoso & Early & + & + & - & - & - & - & - & - \\
\hline Funtumia elastica & Early & - & - & - & + & - & + & - & + \\
\hline Glyphaea brevis & Late & - & - & - & - & - & - & + & - \\
\hline Icacina trichantha & Late & - & + & - & + & - & - & - & - \\
\hline Lecaniodiscus cupanioides & Late & - & - & - & + & - & - & - & + \\
\hline Mallotus oppositifolius & Late & - & + & - & - & - & - & - & - \\
\hline Microdesmis puberula & Late & - & - & - & + & - & - & - & + \\
\hline Napoleona imperialis & Late & - & - & - & + & - & - & - & - \\
\hline Napoleona vogelii & Late & - & - & - & - & - & - & - & + \\
\hline Newbouldia laevis & Early & - & + & + & - & - & - & - & + \\
\hline Olax sp. & Late & - & - & - & - & - & + & - & - \\
\hline Petivera alliacea & Undetermined & - & + & - & - & - & - & - & - \\
\hline Phyllanthus muellerianus & Undetermined & - & - & + & - & - & - & - & - \\
\hline Solanum erianthum & Undetermined & + & - & - & - & - & - & - & - \\
\hline Spondias mombin & Late & - & + & - & - & - & - & - & - \\
\hline Trema orientalis & Early & + & - & - & - & - & - & - & - \\
\hline Trichilia prieureana & Early & - & - & - & - & - & - & - & + \\
\hline Trilepsium madagascariensis & Early & - & - & - & - & - & - & - & + \\
\hline
\end{tabular}

Structural characteristics: density, diversity and evenness of the plots

There was no clear trend of diversity of woody species with the age of the plots. The oldest plot (plot VIII) had the highest individual stem density per hectare (Table 3) while some other older plots (plot IV, VI and VII) had the lowest densities of woody species (Table 3). Some plant species were dominant in terms of their densities in the plots (Table 5). 
Table 5. Relative abundance of dominant species in the different study plots in the Biological Gardens of Obafemi Awolowo University, Ile-Ife, Nigeria

\begin{tabular}{|c|c|c|c|c|c|c|c|c|c|}
\hline \multirow{2}{*}{ Species name } & \multirow{2}{*}{ Family } & \multicolumn{8}{|c|}{ Plot (\% relative abundance) } \\
\hline & & $\mathrm{I}$ & II & III & IV & $\mathrm{V}$ & VI & VII & VIII \\
\hline Albizia lebbeck & Fabaceae & - & - & - & - & - & - & 16 & - \\
\hline Albizia zygia & Fabaceae & - & 10 & 6 & - & - & - & - & - \\
\hline Alchornea cordifolia & Euphorbiaceae & - & 17 & - & - & - & - & - & - \\
\hline Alchornea laxiflora & Euphorbiaceae & - & - & - & - & - & 8 & - & 33 \\
\hline Alstonia boonei & Apocynaceae & - & - & - & - & - & 6 & - & - \\
\hline Blighia sapida & Sapindaceae & - & 13 & - & 5 & - & 6 & - & 3 \\
\hline Carpolobia lutea & Polygalaceae & - & - & - & 4 & - & - & - & 3 \\
\hline Celtis zenkeri & Ulmaceae & - & - & - & 4 & - & - & 10 & - \\
\hline Cnestis ferruginea & Connaraceae & - & - & - & 5 & - & - & - & - \\
\hline Cola millenii & Sterculiaceae & - & - & - & 7 & 9 & 10 & - & - \\
\hline Deinbollia pinnata & Sapindaceae & - & 5 & - & 4 & - & - & - & 6 \\
\hline Ficus exasperata & Moraceae & 8 & - & - & - & - & - & - & - \\
\hline Ficus тисоso & Moraceae & 17 & 5 & - & - & - & - & - & - \\
\hline Funtumia elastica & Apocynaceae & - & - & - & 5 & - & 14 & - & 5 \\
\hline Glyphae brevis & Tiliaceae & - & - & - & - & - & - & 10 & - \\
\hline Icacina tricantha & Icacinaceae & - & 4 & - & 7 & - & - & - & - \\
\hline Lecaniodiscus cupanioides & Sapindaceae & - & - & - & 4 & - & - & - & 6 \\
\hline Mallotus oppositifolius & Euphorbiaceae & - & 4 & - & - & - & - & - & - \\
\hline Microdesmis puberula & Pandaceae & - & - & - & 6 & - & - & - & 8 \\
\hline Napoleona imperialis & Lecythidaceae & - & - & - & 7 & - & - & - & - \\
\hline Napoleona vogelii & Lecythidaceae & - & - & - & - & - & - & - & 3 \\
\hline Newbouldia laevis & Bignoniaceae & - & 6 & 10 & - & - & - & - & 6 \\
\hline Olax sp. & Olacaceae & - & - & - & - & - & 6 & - & - \\
\hline Petivera alliacea & Phytolaccaceae & - & 8 & - & - & - & - & - & - \\
\hline Phyllantus muellerianus & Euphorbiaceae & - & - & 8 & - & - & - & - & - \\
\hline Solanum erianthum & Solanaceae & 14 & - & - & - & - & - & - & - \\
\hline Spondias mombin & Anarcadiaceae & - & 5 & - & - & - & - & - & - \\
\hline Trema orientalis & Ulmaceae & 23 & - & - & - & - & - & - & - \\
\hline Trichilia prieureana & Meliaceae & - & - & - & - & - & - & - & 3 \\
\hline Trilepsium madagascariensis & Moraceae & - & - & - & - & - & - & - & 5 \\
\hline Total & & 62 & 77 & 24 & 58 & 9 & 50 & 36 & 81 \\
\hline
\end{tabular}

The diversity of plant species in the plots as measured by the Shannon-Weiner diversity index in relation to the ages of the plots did not follow a clear trend (Table 3). The diversity was lowest in the youngest plot (plot I) and highest in one of the older plots (plot IV) (Table 3). However, the oldest plot (plot VIII) did not have the highest diversity index (Table 3 ).

The species evenness showed that the species were least evenly distributed in the youngest plot (plot I) and oldest plot (VIII) (Table 3).

\section{Similarity of the plots}

The Sørensen index of similarity showed that plots of the same ages (i.e., plot III, IV, V, VI, VII) had high similarity among them in their species composition. However, plots of different ages, especially plots I and II, had the lowest similarity in species composition with other plots which are also of different ages with them (Table 6). Also, the youngest plot (plot I), when compared with the unburnt plot (plot VIII), had the least similarity in terms of species composition (0.21). When compared with the unburnt plot, plot V had the highest similarity index of 0.38 (Table 6).

\section{Discussion}

The result of this study showed that species composition did not increase with age although the oldest plot (plot VIII) had the highest species composition. This does not agree with the findings of Chazdon et al. (2005), who stated that younger forests (less than 15 years since abandonment) are certainly more dynamic in overall stem density and community sizes than older sites. However, Chazdon et al. (2007) opined that stem density does not show a predictable pattern with stand age. The dominance of Trema orientalis (L.) Blume, an early successional species, in the youngest plot (plot I) shows that the plot is still undergoing some early stage of colonisation. This is in line with the assertion 
of Fantini et al. (2011) who opined that during the early stages of secondary succession, species such as Trema micrantha (L.) Blume have high densities and dominance when they occur. There was also an abundance of some species in the older plots, such as Deinbollia pinnata (Poir.) Schum. \& Thonn., Icacina trichantha Oliv., Lecaniodiscus cupanioides Planch. and Microdesmis puberula Hook. f. ex Planch. (Table 5). This suggests that these species make up part of the late successional stage of a forest. Furthermore, the older plots had a mixture of late and early successional species. This suggests that the plots are gradually tending towards maturity. Thus given more years of recovery, the forest would be replaced with tree species that are characteristic of a climax community.

It was observed that the young plots undergoing early successional phase of regeneration had a high presence of grasses, climbers and herbs . These plots are still undergoing an early building phase of regeneration. This is in line with the reports of Lang \& Knight (1983) and Finegan (1996) that the first decade of forest succession after site abandonment in lowland moist and wet neotropics is characterised by vegetation dominated by grasses, shrubs and forbs. Oliver \& Larson (1990) termed this phase the stand initiation phase. The reason why the oldest selected plot (plot II) did not have the highest number of species could be attributed to succession, during which early colonisers are lost leading to a subsequent drop in the species composition of the next seral stage. This is in line with the conclusion of Eggeling (1947) in Budongo forest, Uganda, that there was an initial rise in species numbers (species density) during succession, reaching a peak at intermediate phases of succession, followed by a decline during late succession. A similar result was reported by Aweto (1981) in a Nigerian moist evergreen forest fallow, where tree density increased over 7 years but stabilised after 10 years.

Species diversity and evenness of species did not follow a clear trend with age of plots in this study. This may be because of the dominance of some early successional species in some of the plots. For example, Trema orientalis dominated the species composition in plot I. The youngest plot (plot I) had the lowest diversity in this study. This suggests that as the forest develops, new species begin to colonise and recruit, leading to a gradual accumulation of species over time (Chazdon et al., 2007).

Some studies have documented an increasing diversity with forest age. For example, Aide et al. (1995) found a positive relationship between stand age and species density in a 60-year chronosequence in Puerto Rico, and Saldarriaga et al. (1988) found a similar Shannon diversity in a 40- year old secondary forest and old growth in very small plots in Colombia and Venezuela. At low level disturbance, the diversity is low because only the best competitors persist. Thus, a peak in diversity should occur at intermediate intensities and frequencies of disturbance, as well as intermediate time since the last disturbance. Fantini et al. (2011) reported an increase in diversity and especially species richness over time since abandonment. Similar cases have been found in other studies of swidden fields, abandoned plantations and pasture (Tabarelli \& Mantovani, 1999; Mitche et al., 2000; Oliveira, 2002). Furthermore, Toky \& Ramakrishnan (1983) reported that in swidden fallow succession in northeastern India, species diversity increased with fallow age during the first 15-20 years. Relationships between relative abundance of species and stand age differed among species, indicating that species achieved their highest abundance at different times during succession as proposed in the model of succession by Gómez-Pompa \& Vázquez-Yanes (1981) and Finegan (1984). This is evident in the presence of more early successional tree and grass species in the youngest plot (plot I). Also, the observation in this study showed that older plots had the highest diversity which is in line with the assertion of Fantini et al. (2011), who stated that species diversity is often greatest during mid-successional stages that contain both early and late successional species.

Table 6. Summary of the Sørensen's co-efficient of similarity between the plots

\begin{tabular}{cccccccccc}
\hline Plot & I & II & III & IV & V & VI & VII & VIII \\
\hline I & - & 0.33 & 0.23 & 0.24 & 0.13 & 0.23 & 0.13 & 0.21 \\
II & 0.66 & - & 0.30 & 0.33 & 0.35 & 0.28 & 0.19 & 0.30 \\
III & 0.77 & 0.70 & - & 0.38 & 0.46 & 0.41 & 0.29 & 0.32 \\
IV & 0.76 & 0.67 & 0.62 & - & 0.53 & 0.37 & 0.33 & 0.36 \\
V & 0.87 & 0.65 & 0.54 & 0.47 & - & 0.36 & 0.32 & 0.38 \\
VI & 0.77 & 0.72 & 0.59 & 0.63 & 0.64 & - & 0.30 & 0.25 \\
VII & 0.88 & 0.81 & 0.71 & 0.67 & 0.68 & 0.70 & - & 0.26 \\
VIII & 0.79 & 0.70 & 0.68 & 0.64 & 0.62 & $0 .+$ & 0.74 & - \\
\hline
\end{tabular}

Note: Upper right represent similarity index between study plots and lower left represent dissimilarity index between study plots. 
The evenness of distribution of species in this study was the least in the youngest plot but high in the older plots. This could be because the younger plot was still in the preliminary phase of colonisation whereby newer species are still arriving, whereas the older plots have attained some level of stability and tending towards another successional phase. This supports the assertion of Capers et al. (2005) who reported that evenness of species appear to increase for at least 30 years after pasture abandonment in wet tropical forests in Costa Rica. They attributed this to an increase in tree species as a proportion of all species with successional age and also because the proportion of rare tree species was higher in the oldest site than in the youngest. This could be the reason why the plots of similar ages had more evenness pattern compared to those of different ages in this study.

The comparison of old and young plots using Sørensen's index of similarity showed that the youngest plot had the least similarity with the unburnt plot as against the comparison made between the older plots and the unburnt plot. This implies that the young plot must pass through some seral stages like the other plots to attain climax (Horn, 1981).

\section{Conclusions and Recommendations}

The result of this study showed that plots of similar ages had higher similarities in species composition than those that were different in age. Thus, as species tend to converge in forests per seral stage, similarity increases provided they are of similar ages. This study also showed that the youngest plot had the least species diversity and evenness of distribution. Also, species diversity, composition and evenness of distribution were not dependent on stand age. Thus, this study (although conducted on a small area) can serve as a means of predicting how a forest that has been disturbed would look like, per seral stage, in terms of species composition and diversity, most especially in the West African zone.

Even though the biological gardens of Obafemi Awolowo University campus is not listed as part of the protected areas in Nigeria, the results of this study highlight the need for the establishment of the forest as a regional Protected Area. Even given its relatively small size, it is a forest with minimal human disturbance and has a high value for biodiversity management and conservation. By this, future successional monitoring studies which will check the recovery of the forest can be conducted.

\section{References}

Ademoh F.O., Muoghalu J.I., Onwumere B. 2017. Temporal pattern of tree community dynamics in a secondary forest in southwestern Nigeria, 29 years after a ground fire. Global Ecology and Conservation 9: 148-170. DOI: 10.1016/j.gecco.2016.11.005

Aide T.M., Zimmermann J.K., Herrera L., Rosario M., Serrano M. 1995. Forest recovery in abandoned tropical pastures in Puerto Rico. Forest Ecology and Management 77: 77-86. DOI: 10.1016/03781127(95)03576-V

Allaby M. 2010. The Concise Oxford Dictionary of Ecology, fourth ed. Oxford: Oxford University Press. 416 p.

Aweto A.O. 1981. Secondary succession and soil fertility restoration in South-Western Nigeria I, Succession. Journal of Ecology 69: 601-607. DOI: 10.2307/2259686

Ayodele O.J. 1986. Phosphorus availability in savanna soils of Western Nigeria. Tropical Agriculture (Trinidad) 63: 297-300.

Capers R.S., Chazdon R.L., Brenes A.R., Alvarado B.V. 2005. Successional dynamics of woody seedling communities in wet tropical secondary forests. Journal of Ecology 93: 1071-1084. DOI: 10.1111/j.13652745.2005.01050.x

Charter J.R. 1969. Map of Ecological zones of Nigerian Vegetation. Ibadan: Federal Department of Forestry.

Chazdon R.L. 2003. Tropical forest recovery: Legacies of human impact and natural disturbances. Perspectives in Plant Ecology, Evolution and Systematics 6: 51-71. DOI: 10.1078/1433-8319-00042

Chazdon R.L. 2008. Chance and determinism in tropical forest succession. In: W.P. Carson, S.A. Schnitzer (Eds.): Tropical Forest Community Ecology. Wiley-Blackwell Publishing. P. 384-408.

Chazdon R.L., Redondo Brenes A., Alvarado B.V. 2005. Effects of climate and stand age on annual tree dynamics in tropical second-growth rain forests. Ecology 86: 1808-1815. DOI: 10.1890/04-0572

Chazdon R.L., Letcher S., Breugel M., van MartinezRamos M., Bongers F., Finegan B. 2007. Rates of change in tree communities of secondary tropical forests following major disturbances. Proceedings of the Royal Society of London, Series B 362: 273-289. DOI: $10.1098 /$ rstb.2006.1990

Chesson P., Huntly N. 1997. The roles of harsh and fluctuating conditions in the dynamics of ecological communities. American Naturalist 150: 519-553. DOI: 10.1086/286080

Connell J.H. 1978. Diversity in tropical rainforests and coral reefs. Science 199: 1302-1310. DOI: 10.1126/science.199.4335.1302

Denslow J.S., Ellison A., Sanford R.E. 1998. Treefall gap size effects on ecological processes in a tropical wet forest. Journal of Ecology 86: 591-609. DOI: 10.1046/j.13652745.1998.00295.x 
Dupuy J.M., Chazdon R.L. 2006. Effects of vegetation cover on seedling and sapling dynamics in secondary tropical wet forests in Costa Rica. Journal of Tropical Ecology 22: 1-22. DOI: 10.1017/ S0266467405002890

Eggeling W.J. 1947. Observations on the ecology of the Budongo rainforest, Uganda. Journal of Ecology 34: 20-87. DOI: $10.2307 / 2256760$

Fantini A.C., Siminski A., Guries R.P., Ruschel A.R., dos Reis M.S. 2011. Secondary forest succession in the Mata Atlantica, Brazil: Floristic and Phytosociological Trends. ISRN Ecology 2011: 1-20. DOI: $10.5402 / 2011 / 759893$

FAO/UNESCO. 1974. Legend to Soil Map of the World. Vol.1. World soil classification. Paris: UNESCO. 59 p.

Finegan B. 1984. Forest succession. Nature 312: 109-114. DOI: $10.1038 / 315692 \mathrm{a} 0$

Finegan B. 1996. Pattern and process in neotropical secondary forests: the first 100 years of succession. Trends in Ecology and Evolution 11: 119-124. DOI: 10.1016/0169-5347(96)81090-1

Gómez-Pompa A., Vázquez-Yanes C. 1981. Successional studies of a rain forest in Mexico. In: D.C. West, H.H. Shugart, D.B. Botkin (Eds.): Forest Succession: Concepts and Application. NewYork: Springer-Verlag. P. 246-266.

Guariguata M., Ostertag R. 2001. Neotropical secondary forest succession: changes in structural and functional characteristics. Forest Ecology and Management 148: 185206. 10.1016/S0378-1127(00)00535-1

Horn H.S. 1981. Some causes of variety in pattern of secondary succession. In: D.C. West, H.H. Shugart, D.B. Botkin (Ed.): Forest Succession: Concepts and Application. NewYork: Springer-Verlag. P. 24-35.

Isichei A.O., Ekeleme F., Jimoh B.A. 1986. Changes in a secondary forest in South Western Nigeria following a ground fire. Journal of Tropical Ecology 2: 249-256. DOI: 10.1017/S0266467400000870

Keay R.W.J. 1959. An Outline of Nigerian Vegetation. $3^{\text {rd }}$ edition. Lagos: Federal Ministry of Information, Printing Division. $46 \mathrm{p}$.

Lang G.E., Knight D.H. 1983. Tree growth, mortality, recruitment, and canopy gap formation during a 10-year period in a tropical moist forest. Ecology 64: 10751080. DOI: $10.2307 / 1937816$

LauranceW.F.,FerreiraL.V.,Rankin-DeMerinaJ.M.,Laurance S.G. 1998. Rainforest fragmentation and the dynamics of the Amazonian tree communities. Ecology 79: 2032 2040. DOI: 10.1890/0012-9658(1998)079[2032:RFFA TD]2.0.CO;2

Mitche T., Zimmerman J.K., Pascarella J.B., Rivera L., Marcano-Vega H. 2000. Forest regeneration in a chronosequence of tropical abandoned pastures: implications for restoration ecology. Restoration Ecology 8(4): 328 338. DOI: 10.1046/j.1526-100x.2000.80048.x

Noss R.S. 1996. Conservation of biodiversity at the landscape scale. In: R.C. Szaro, D.W. Johnston (Eds.): Biodiversity in Managed Landscapes: Theory and Practice. USA: Oxford. P. 574-589.

Ogunniran O., Muoghalu J.I. 2007. Vegetation types and composition in altitudinal gradient on an inselberg at Ile-Ife, Nigeria. Nigerian Journal of Botany 20: 9-44.

Oliveira R.R. 2002. Ação antrópica e resultantes sobre a estrutura e composição da Mata Atlântica na Ilha Grande, R.J. Rodriguesia 53 (82): 33-58.

Oliver C.D., Larson B.C. 1990. Forest Stand Dynamics. NewYork: McGraw-Hill. 467 p.

Saldarriaga J.G., West D.C., Tharp M.L., Uhl C. 1988. Longterm chronosequence of forest succession in the upper Rio Negro of Colombia and Venezuela. Journal of Ecology 76: 938-958. DOI: 10.2307/2260625

Sheil D., Burslem D. 2003. Disturbing hypotheses in tropical forests. Trends in Ecology and Evolution 18: 18-26. DOI: 10.1016/S0169-5347(02)00005-8

Tabarelli M., Mantovani W.A. 1999. A regeneração de uma floresta tropical montana após corte e queima (São Paulo - Brasil). Revista Brasileira de Biologia 59 (2): 239250. DOI: $10.1590 / \mathrm{S} 0034-71081999000200008$

Toky O.P., Ramakrishnan P.S. 1983. Secondary succession following slash and burn agriculture in north-eastern India. I. Biomass, litterfall, and productivity. Journal of Ecology 71: 735-745. DOI: 10.2307/2259589

USDA. 1975. Soil Taxonomy, Agriculture Handbook. Washington: U.S. Department of Agriculture.

Van Gemerden B.S., Shu G.N., Olff H. 2003. Recovery of conservation values in Central African rain forest after logging and shifting cultivation. Biodiversity and Conservation 12: 1553-1570. DOI: 10.1023/A:1023603813163

Walker L.R., Chapin F.S.I. 1987. Interactions among processes controlling successional change. Oikos 50: 131-135.

White F. 1983. The Vegetation of Africa. A descriptive memoir to accompany map of Africa. Paris: UNESCO. 356 p. 


\title{
ВИДОВОЕ РАЗНООБРАЗИЕ И ДИНАМИКА СУКЦЕССИИ ВТОРИЧНОГО ЛЕСА БИОЛОГИЧЕСКИХ САДОВ УНИВЕРСИТЕТА ОБАФЕМИ АВОЛАУ, ИЛЕ-ИФЕ, НИГЕРИЯ
}

\author{
Н. О. Увалака", Дж. И. Муогалу, А. О. Осеволе \\ Университет Обафеми Аволау, Нигерия \\ *e-mail: uwalakanelson@gmail.com
}

\begin{abstract}
Во всем мире леса постоянно нарушаются в результате воздействий человека и естественных событий, а нарушение тропических лесов особенно выражено. Об этом свидетельствует низкий уровень их биоразнообразия. В этом исследовании изучались флористический состав, видовое разнообразие

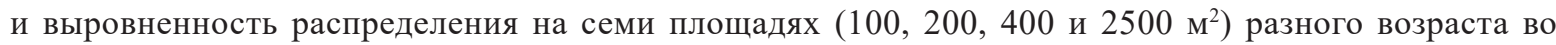
вторичном лесу Биологического сада Университета Обафеми Аволау, Иле-Ифе. Целью этого исследования было определить изменения этих параметров в ходе сукцессии. На каждом участке были учтены и перечислены все виды сосудистых растений. Собранные данные были использованы для определения флористического состава, плотности, разнообразия и выровненности видов растений в лесу. В общей сложности выявлено 85 видов древесных растений (69\%), 3 травянистых растения (2\%), 13 полукустарников (11\%) и 21 лиана (17\%). Они относятся к 113 родам и 52 семействам. Количество видов древесных растений на участках изменялось в зависимости от возраста участков. Однако четкой связи плотности и разнообразия видов с возрастом сукцессии не выявлено. В лесу доминировали виды ранних этапов сукцессии (50\%) против $33 \%$ видов поздних этапов сукцессии. Кроме того, на самом молодом участке (Участок I) преобладали виды ранних этапов сукцессии, в то время как на более старых участках преобладала смесь видов как ранних так и поздних этапов сукцессии. Сходство флористического состава между площадями в течение сукцессии в тропическом лесу было выше между участками одного и того же возраста. Кроме того, это исследование показало, что из-за направленной картины сукцессии, виды, которые составляют разнообразие лесов разного возраста, могут быть использованы для предсказания путей их восстановления.
\end{abstract}

Ключевые слова: виды сукцессии, вторичный лес, выровненность, разнообразие, растительная сукцессия, сходство 